\title{
Trends in epilepsy surgery: stable surgical numbers despite increasing presurgical volumes
}

\author{
Thomas Cloppenborg, ${ }^{1}$ Theodor W May, ${ }^{1}$ Ingmar Blümcke, ${ }^{2}$ Philip Grewe, ${ }^{1}$ \\ Lena J Hopf, ${ }^{1}$ Thilo Kalbhenn, ${ }^{3}$ Margarete Pfäfflin, ${ }^{1}$ Tilman Polster, ${ }^{1}$ Reinhard Schulz, ${ }^{1}$ \\ Friedrich G Woermann, ${ }^{1}$ Christian G Bien ${ }^{1}$
}

\begin{abstract}
- Additional material is published online only. To view please visit the journal online (http://dx.doi.org/10.1136/ jnnp-2016-313831).

${ }^{1}$ Bethel Epilepsy Centre, Bielefeld, Germany ${ }^{2}$ Institute of Neuropathology, University of Erlangen, Erlangen, Germany ${ }^{3}$ Department of Neurosurgery, Evangelisches Krankenhaus, Bielefeld, Germany
\end{abstract}

\section{Correspondence to} Dr Thomas Cloppenborg, Bethel Epilepsy Centre, Maraweg 21, Bielefeld D-33617, Germany; thomas.cloppenborg@mara.de

Received 21 April 2016 Revised 22 July 2016 Accepted 7 September 2016 Published Online First 5 October 2016 2016;87:1322-1329.

\section{ABSTRACT}

Introduction Despite the success of epilepsy surgery, recent reports suggest a decline in surgical numbers. We tested these trends in our cohort to elucidate potential reasons.

Patients and methods Presurgical, surgical and postsurgical data of all patients undergoing presurgical evaluation in between 1990 and 2013 were retrospectively analysed. Patients were grouped according to the underlying pathology.

Results A total of 3060 patients were presurgically studied, and resective surgery was performed in $66.8 \%$ $(n=2044)$ of them: medial temporal sclerosis (MTS): $n=675,33.0 \%$; benign tumour (BT): $n=408,20.0 \%$; and focal cortical dysplasia (FCD): $n=284,13.9 \%$. Of these, 1929 patients (94.4\%) had a follow-up of 2 years, and $50.8 \%$ were completely seizure free (Engel IA). Seizure freedom rate slightly improved over time. Presurgical evaluations continuously increased, whereas surgical interventions did not. Numbers for MTS, BT and temporal lobe resections decreased since 2009. The number of non-lesional patients and the need for intracranial recordings increased. More evaluated patients did not undergo surgery (more than $50 \%$ in 2010-2013) because patients were not suitable (mainly due to missing hypothesis: $4.5 \%$ in $1990-1993$ up to $21.1 \%$ in $2010-2013$, total $13.4 \%$ ) or declined from surgery (maximum $21.0 \%$ in $2010-2013$, total $10.9 \%$ ). One potential reason may be that increasingly detailed information on chances and risks were given over time. Conclusions The increasing volume of the presurgical programme largely compensates for decreasing numbers of surgically remediable syndromes and a growing rate of informed choice against epilepsy surgery. Although comprehensive diagnostic evaluation is offered to a larger group of epilepsy patients, surgical numbers remain stable.

\section{INTRODUCTION}

Epilepsy surgery has become a standard treatment option for patients with intractable focal epilepsy. A recent Cochrane review identified 177 studies reporting on surgical outcome of more than 16000 patients. ${ }^{1}$ In total, $65 \%$ of the patients had a good outcome, defined as seizure freedom, usually apart from auras (range across studies from $13.5 \%$ to $92.5 \%)$. For temporal lobe epilepsy, two randomised controlled trials demonstrated the superiority of resection in comparison to medical treatment. ${ }^{2} 3$ Therefore, international guidelines recommend an early referral of patients with drug-resistant seizures for comprehensive evaluation including surgical options. ${ }^{45}$

Despite this success of epilepsy surgery, reports from Europe and the USA suggest a decline in resective surgeries over the past decade. ${ }^{6-11}$

A decreasing proportion of patients with medial temporal lobe epilepsy and an increasing complexity of cases have been suggested as underlying reasons. ${ }^{6} 91112$

The Epilepsy Centre Bethel has been one of the surgically most active centres in Germany for more than 20 years. We analysed the Bethel series to verify the above-mentioned trends in epilepsy surgery. We intended to further elaborate reasons for a potentially non-increasing proportion of patients proceeding to resective surgery. We hypothesised that (1) the increasing number of 'difficult cases' leads to a larger number of patients without sufficient focus hypothesis or with inacceptable surgical risks and that (2) an increasing awareness of chances and risks may influence patient's decision on surgery.

\section{MATERIALS AND METHODS}

Retrospective data evaluation

The principles of presurgical evaluation and decision-making have been constant since initiation of the programme in cooperation with the Cleveland Clinic under H.O. Lüders in 1990. The evaluation aims at the delineation of the epileptogenic zone and the judgement on its removal without inacceptable deficits. ${ }^{13}$

Routine presurgical evaluation included (1) video-EEG monitoring and MRI in order to formulate a hypothesis for the epileptogenic zone and (2) investigations for risk stratification including neuropsychological testing and fMRI-based language lateralisation. MRI field strength increased over time: 1990-2000 external <1.0 T, 2000-2010 internal $1.5 \mathrm{~T}$ and since 2011 internal 3.0 T. Additional imaging techniques such as FDG-PET, DTI fibretracking and invasive language lateralisation by intracarotid amobarbital test (Wada test) were performed as indicated. A case conference was held only for patients with decision on operability and the need of intracranial recordings. Protocols of these case conferences and additional investigations for presurgical risk stratification were used to identify all potential surgical candidates. Patients with other indication for video-EEG recording (eg, for differential diagnosis) were excluded by this selection. 
The study was conducted in accordance with the Gesundheitsdatenschutzgesetz (GDSG NRW, German law of healthcare data protection). Data were obtained from the Bethel presurgical/surgical epilepsy database (status as of 17 December 2015), updated with presurgical, surgical and postsurgical data by PG and TM.

For patients with more than one evaluation or surgical intervention, the latest was used for this analysis.

\section{Grouping according to pathology}

Neuropathological diagnosis was performed by the Bethel neuropathological institute from 1990 to 2011, thereafter by the neuropathological institute of the University of Erlangen. Until 2011, localised malformations of cortical development were classified as focal cortical dysplasias (FCDs) according to Palmini. ${ }^{14}$ These results were transferred to the recent classification system of the International League against Epilepsy. ${ }^{15}$ Type I dysplasias were not further disentangled. Differentiation of type IIa and IIb dysplasias depended on the detection of dysmorphic neurons for type IIa and balloon cells for type IIb, respectively.

Dual pathology was defined as the coexistence of medial temporal sclerosis (MTS) and another epilepsy related pathology: (1) malformation of cortical development, (2) tumour, (3) phacomatosis, (4) vascular, (5) encephalitis and (6) gliosis due to perinatal injury or trauma. Gliosis of unspecified reasons and mild forms of cortical malformation in patients with MTS $^{16}{ }^{17}$ were not counted for the diagnosis of dual pathology. MTS in the context of hemispheric pathologies was interpreted as element of the main pathology and not classified as dual pathology.

Patients were categorised according to histopathology diagnosis or-if no operation took place-by presurgical MRI. In some patients, MRI and pathology findings had to be integrated to come to a meaningful diagnosis. ${ }^{18}$ The groups were arranged according to Engel as 'surgical remediable syndromes' (like MTS, benign tumour (BT), FCD and the following diffuse hemispheric pathologies (DH): Rasmussen's encephalitis, Sturge-Weber syndrome, infarctions of one medial cerebral artery, unilateral porencephaly and hemimegalencephaly) and 'potential candidates' (like dual pathology, tuberous sclerosis or non-lesional patients). ${ }^{19}$

\section{Presurgical protocol and surgery types}

Usually, resections tightly restricted to the detectable lesions were performed. In the 2010-2013 period, extended lesionectomies were regularly performed if no standard resection were offered (publications had demonstrated the benefit of complete and-if by any means possible-extended removal of FCDs ${ }^{20}{ }^{21}$ ). In selected cases, lesionectomy was guided by intracranial recordings and electrocorticography. The anteromedial two-thirds of the temporal lobe were resected in almost all patients with MTS. Patients with temporo-apical lesions sparing the hippocampus received an apical temporal lobe resection without removal of the hippocampus but including the amygdala. ${ }^{22}$ Patients with DH pathologies underwent hemispherectomy in one of its modern 'functional', that is, partly disconnective variants, mainly by removal of the anterior temporal lobe and insular cortex and sectioning of the corpus callosum.

\section{Patients' surgical outcome}

All patients were actively invited for postoperative visits 6 and 24 months after surgery including routine postoperative MRI after 6 months, EEG and neuropsychological testing as well as social follow-up at both visits. Documentation of seizure freedom regarding to Engel's classification was performed at both timepoints. ${ }^{23}$ For this study, outcome data 24 months after operation were used because changes in seizure frequency before this time-period occur frequently. ${ }^{24}$ Seizure freedom was rigorously defined as 'no seizures and no auras since discharge from the hospital after surgery', that is, Engel class IA $^{23}$ for reasons detailed recently ${ }^{10}$ and to avoid inconsistencies across studies due to different outcome scores. ${ }^{1} 25$

\section{'Intention-to-treat' seizure freedom rates}

We calculated the percentage of postsurgical seizure-free patients as a proportion of all presurgically evaluated patients ('intention-to-treat ratio' (sf(ITT)). ${ }^{10}$ This ratio reflects the 'yield' of an epilepsy programme and makes the comparison between series from different centres more meaningful. For example, a selective attitude may result in better rates of postsurgically seizure-free patients but will be reflected in a lower sf(ITT).

\section{Decision against surgery}

The case conference formulated an individual decision on operability. A decision in favour of surgery was usually made for patients with a sufficiently high chance for postoperative seizure freedom and an acceptable risk for postoperative deficits. In some cases like patients with Rasmussen's encephalitis, the high chance for postoperative seizure freedom and the high seizure burden of the patients may even justify postoperative hemiparesis and hemianopia. ${ }^{26}$

\section{Expert's reasons against surgery}

Reasons for negative decisions on operability have been (1) the missing identification of a circumscribed epileptogenic zone and (2) an inacceptable risk for postoperative deficits like aphasia, hemiparesis, visual field deficits or memory decline. These risks were extracted from the case conference protocols or judged by the location of the epileptogenic lesion and its neighbourhood to eloquent cortex. In patients with multiple risks, the one with the most severe impact on functions of daily life was noted (graded severity as in the list given above).

\section{Patient's reasons against surgery}

Some patients were offered surgery subsequent to the case conference but opted against this option. We identified these patients by the missing surgical intervention despite positive decision on operability. To test the hypothesis of increasingly detailed informed consent procedures as a measurable influence on patients' decision, we reviewed the informed consent protocols of 69 patients with left temporal lobe resection because of MTS (because of the assumed homogenous risk profile). For each year from 1990 to 2013, the first three operated patients were selected. We assessed the existence of a written consent, document length, the number of risks mentioned and the existence of an individual sketch of the proposed procedure (as an indicator for the intensity of the information).

\section{Statistical analyses}

For trend analyses, patients were grouped into four yearperiods. $\chi^{2}$ Test was performed to test the homogeneity of proportions of operated patients over the periods, and Mantel-Haenszel $\chi^{2}$ test of linear trend was used to test whether there was a (linear) trend of the proportions of operated patients over time. In addition, generalised linear models (GLMs) including the Bonferroni adjusted post hoc tests were performed to identify those periods that deviate significantly from the estimated total mean. 
Statistical analyses were performed using the IBM SPSS Statistics for Windows (V.23.0; Armonk, New York, USA: IBM Corp).

\section{RESULTS}

\section{Presurgical evaluation, surgery and outcome}

Table 1 and online supplementary table S1 give demographic data, decision on operability, resective surgeries and outcome 24 months after surgery.

A total number of 3060 patients (age, mean \pm SD: 25 \pm 16 years, range: $0-74$ years; women: $47.2 \%$ ) were presurgically studied. Of all evaluated patients, 64.9\% $(n=1985)$ had a surgically remediable syndrome. ${ }^{19}$ The three most frequent aetiologies were MTS ( $n=767,25.1 \%$ of the evaluated patients), BT $(n=498,16.3 \%)$ and FCD $(n=433,14.2 \%)$. An operative treatment option was offered to $92.3 \%$ of the patients with surgically remediable syndromes and to $48.6 \%$ of the 'potentially remediable syndromes'.

Resective surgery was performed in 2044 patients $(66.8 \%$ of the evaluated patients). A proportion of $76.8 \%$ of the operations was performed in patients with surgically remediable syndromes $(n=1570)$. Most frequent aetiologies again were MTS $(\mathrm{n}=675,33.0 \%)$, BT $(\mathrm{n}=408,20.0 \%)$ and FCD $(\mathrm{n}=284$, $13.9 \%)$. Of these, 192 patients (9.4\%) underwent more than one surgical intervention. Outcome data were available for as many as 1929 patients (94.3\%). The overall seizure freedom rate was $50.8 \%$. BT and cavernomas showed the best response to surgical treatment (seizure freedom rate $60.1 \%$ and $68.3 \%$, respectively). The overall sf(ITT) was $33.9 \%$. 'Surgically remediable syndromes' had a higher sf(ITT) of $42.3 \%$. 'Potential surgical candidates' showed a lower sf(ITT) of $16.6 \%$.

\section{Trends over time}

Presurgical evaluation and surgical treatment

Figure 1 illustrates the number of presurgical evaluations and resective surgeries over time. Further subgroup information is given in online supplementary table S2.
Presurgical evaluations increased continuously over time. The number of surgical interventions rose up until 2002-2005 ( $n=421$ resections) and was slightly decreasing in the following years (down to 2010-2013: $n=326$ resections). The resulting trend of an increasing number of evaluated patients finally not undergoing surgery is illustrated in figure 2 . The total proportion of operated patients was $66.8 \%$. Whereas in the first and third periods, the proportions were significantly higher than the mean, in the last period of 2010-2013, significantly fewer patients have undergone surgery. For the first time, $<50 \%$ of the evaluated patients were operated on.

There were two tendencies behind this: first, the more 'straightforward' cases within the presurgical cohort show a non-linear (inverted U-shaped) curve similar to the resected patient group. This can be seen in different patient subgroups.

For the two most frequent, usually easy-to-decide-on pathologies, MTS and BT, the number of evaluated patients and consecutive resections showed a non-linear curve similar to the total operated cohort. The percentage of consecutive resection declined over time (figure 3A, B). Resections within the temporal lobe, considered to be less difficult to decide on, showed an inverted U-shaped curve, too (mean 65.1\%, maximum 2002-2005: 71.8\%, minimum 2010-2013: 53.2\%, figure 3C). In contrast, for the suggested intracranial recordings and for non-lesional patients, the curve was $U$ shaped: for invasive recordings, the proportion was high at the beginning of the programme (1990-1993: 40\% of all evaluated patients), dropped down to $5.8 \%$ in $2002-2005$ and rose up to $20.5 \%$ in the last period; for non-lesional patients the minimum percentage was $4.4 \%$ in $2002-2005$ and rose up to $15.5 \%$ (figure $3 \mathrm{D}$ ).

Second, the rate of decisions against surgery is increasing. Figure 4A and online supplementary table S3 show decisions against surgery over time as a proportion of the total cohort of evaluated patients.

There is on the one hand a significant increase of rejection from surgery by the neurologists. In the last period, 2010-

Table 1 Patients' data at presurgical assessment, resective surgery and their outcome 24 months after surgery

\begin{tabular}{|c|c|c|c|c|c|c|c|c|c|c|}
\hline & \multicolumn{6}{|c|}{ Presurgical assessment } & \multirow{2}{*}{$\begin{array}{l}\begin{array}{l}\text { Resective } \\
\text { surgery }\end{array} \\
\\
\mathrm{N}(\% \text { of } \\
\text { presurgically } \\
\text { studied } \\
\text { patients) }\end{array}$} & \multicolumn{3}{|c|}{$\begin{array}{l}\text { Follow-up } 24 \text { months after } \\
\text { surgery }\end{array}$} \\
\hline & $\begin{array}{l}\mathrm{N}(\% \text { of all } \\
\text { presurgically } \\
\text { studied } \\
\text { patients) }\end{array}$ & $\begin{array}{l}\text { Female } \\
(\%)\end{array}$ & $\begin{array}{l}\text { Age at onset } \\
\text { (years) }\end{array}$ & $\begin{array}{l}\text { Epilepsy } \\
\text { duration } \\
\text { (years until } \\
\text { surgery) }\end{array}$ & $\begin{array}{l}\text { Age at } \\
\text { assessment } \\
\text { (years) }\end{array}$ & $\begin{array}{l}\text { Surgical } \\
\text { treatment } \\
\text { offered }(\%)\end{array}$ & & $\begin{array}{l}\text { PO24 } \\
\text { available of } \\
\text { patients } \\
\text { undergoing } \\
\text { resective } \\
\text { surgery (\%) }\end{array}$ & $\begin{array}{l}\text { Seizure } \\
\text { free since } \\
\text { surgery } \\
\text { (Engel } \\
\text { class IA) } \\
(\%)\end{array}$ & $\begin{array}{l}\text { Sf } \\
\text { (ITT) } \\
\text { (\%) }\end{array}$ \\
\hline Total group & $3060(100)$ & 47.2 & $10 \pm 11(0-74)$ & $15 \pm 12(0-60)$ & $25 \pm 16(0-74)$ & 78.2 & $2044(66.8)$ & $1929(94.3)$ & $980(50.8)$ & 33.9 \\
\hline $\begin{array}{l}\text { Surgically remediable } \\
\text { syndromes }\end{array}$ & 1985 (64.9) & 48.8 & $10 \pm 11(0-74)$ & $16 \pm 13(0-59)$ & $25 \pm 16(0-74)$ & 92.3 & $1570(79.1)$ & $1487(94.7)$ & 795 (53.5) & 42.3 \\
\hline MTS & $767(25.1)$ & 54.6 & $11 \pm 10(0-68)$ & $23 \pm 13(1-60)$ & $34 \pm 14(2-71)$ & 97.3 & $675(88.0)$ & $655(97.0)$ & 339 (51.8) & 45.5 \\
\hline $\mathrm{BT}^{*}$ & $498(16.3)$ & 46.8 & $13 \pm 12(0-74)$ & $12 \pm 10(0-50)$ & $25 \pm 14(0-75)$ & 92.2 & $408(81.9)$ & $383(93.6)$ & $230(60.1)$ & 49.2 \\
\hline Cavernoma & $80(2.6)$ & 52.5 & $23 \pm 13(0-55)$ & $11 \pm 10(0-46)$ & $34 \pm 15(6-64)$ & 92.2 & $63(78.8)$ & $60(95.2)$ & $41(68.3)$ & 53.8 \\
\hline FCD (type I+II)* & $433(14.2)$ & 46.2 & $5 \pm 6(0-35)$ & $12 \pm 11(0-49)$ & $18 \pm 14(0-60)$ & 81.1 & $284(65.6)$ & $267(94.0)$ & $122(45.7)$ & 30.0 \\
\hline $\mathrm{DH}^{*}$ & $207(6.8)$ & 39.6 & $4 \pm 8(0-62)$ & $6 \pm 7(0-37)$ & $11 \pm 12(0-60)$ & 81.6 & $140(67.6)$ & $122(87.1)$ & $63(51.6)$ & 34.9 \\
\hline $\begin{array}{l}\text { Potentially remediable } \\
\text { syndrome* }\end{array}$ & $590(19.3)$ & 47.3 & $9 \pm 10(0-50)$ & $15 \pm 13(0-56)$ & $23 \pm 16(0-67)$ & 48.6 & $209(35.4)$ & $200(95.7)$ & $94(47.0)$ & 16.6 \\
\hline Dual pathology & $214(7)$ & 48.1 & $9 \pm 10(0-50)$ & $18 \pm 13(0-58)$ & $27 \pm 17(0-67)$ & 4.2 & $124(57.9)$ & $118(95.2)$ & $63(53.4)$ & 30.9 \\
\hline Non-lesional & $272(8.9)$ & 46.3 & $12 \pm 10(0-47)$ & $15 \pm 11(1-36)$ & $26 \pm 13(0-59)$ & 2.9 & $30(11.0)$ & 29 (96.7) & 8 (27.6) & 3.0 \\
\hline
\end{tabular}

Means \pm SDs, and ranges in parentheses or number and percentages are reported.

${ }^{*}$ For further subgroups, see online supplementary table S1.

BT, benign tumour; DH, diffuse hemispheric; FCD, focal cortical dysplasia; MTS, medial temporal sclerosis; PO24, postoperative follow-up 24 months after surgery; sf(ITT), seizure-free intention to treat rate. 
Figure 1 Trends over time of presurgical evaluation and resective surgeries.
A: total numbers

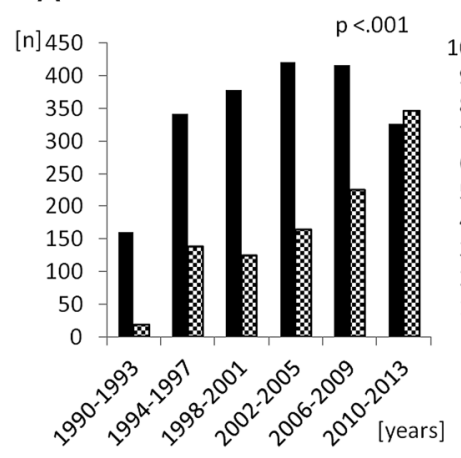

B: proportions in \%

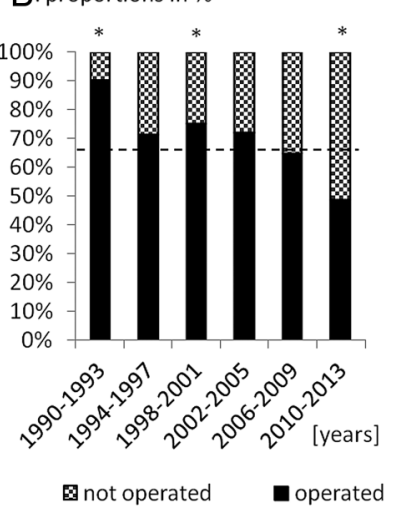

Figure 2 Numbers and proportions of operated and non-operated patients after presurgical evaluation. Legend: test of trend (Mantel-Haenszel $\chi^{2}$ test, $p<0.001$ ) indicates that the number of non-operated patients after presurgical assessment compared to the number of operated patients has increased over time. Dotted line $=$ total (\%). * Significant deviation from estimated grand mean $(p<0.05)$ using GLM. GLM, generalised linear model.

2013 , this rate was $28.7 \%$, the total proportion being $20.1 \%$. Main reason for rejection from surgery was the missing identification of a circumscribed epileptogenic zone (figure 4B) that also showed a highly significant increase over time $(4.5 \%$ in 1990-1993 up to $21.1 \%$ in 2010-2013, total 13.4\%). Expected postoperative deficits as rejection reason were less frequent (6.7\% of all patients) and relatively remained stable over time. Online supplementary figure S1 gives additional information about the distribution of anticipated deficits, which were stable over time (data not shown). The rejection rate by the neurologist for non-lesional patients was constantly high (total $76.8 \%$, $\mathrm{p}=0.281)$.

On the other hand, patients/parents opted increasingly against surgery (figure 4A). This trend reached a maximum in the period of 2010-2013 (21.0\% of all evaluated patients (total $10.9 \%$ ) figure $4 \mathrm{~A}$, online supplementary table S3 and $29.4 \%$ of all rejections from surgery (total $13.7 \%$, data not shown)). This trend was also true for standard aetiologies MTS and BT: the proportion of not operated patients increased (figure $3 \mathrm{~A}, \mathrm{~B}$ ), and more patient/parents opted against surgery (figure 4C, D), for BT a maximum of 30\% in 2010-2013 (figure 4D). To identify a potential reason for this increasing scepticism, 61 informed consent protocols of adult patients with left temporal lobe epilepsy were reviewed for changes of informed consent procedures; for 8 patients, informed consent protocols were not found in the patient files. Table 2 shows that over time, more detailed information on risks and chances were given to the surgical candidates and their families.

The protocols became more extensive (1 page in 1990 and 2010 to 6 pages from the neurosurgeon plus 4 pages from the epileptologist since 2011). More severe complications were mentioned over time, and the expected chance of seizure freedom as per cent (and inversely, of surgical failure) was mentioned only from 1997 on.

\section{Seizure freedom rate}

The overall rate of seizure-free patients 24 months after surgery showed a slight, but significant improvement over time $(\mathrm{p}=0.039$, figure 5). The same was true for BT and especially for FCD, but not for MTS.

\section{DISCUSSION}

In this retrospective single-centre study from Germany, we report on 3060 presurgically evaluated and 2044 operated patients between 1990 and 2013. Patient composition and success rates were similar to other series and identified trends towards more evaluations but not more surgeries because of more complex cases and more reluctance of patients to undergo surgery.

The proportion of patients with 2-year outcomes was exceptionally high $(94.3 \%$, table 1$)$. Other single-centre studies had follow-up data 24 months after surgery from $67.4 \%$ to $85.6 \% .^{10} 2728$ Similar to a single-centre study from Bonn, Germany, ${ }^{10}$ we calculated the sf(ITT). The overall sf(ITT) in our cohort was $33.9 \%$, which is comparable to the Bonn series. This had an sf(ITT) of $32.4 \%$ within 2684 presurgical evaluations, 1721 resective surgeries and 1160 (67.4\%) patients with a follow-up of at least 1 year and a seizure freedom rate (Engel IA) of $50.5 \% .^{10}$

Comparing epilepsy surgery outcome between studies is challenging, because different outcome measures have been used. $^{125}$ We only counted patients completely seizure free since surgery (Engel class IA). This clear definition eliminates arbitrariness in defining 'non-disabling seizures' and 'withdrawal seizures' ${ }^{10}$ and the potential social and prognostic impact of such seizure types. The overall Engel IA seizure freedom rate in our cohort was 50.8\%. Apart from Bonn, the Queen Square series achieved 49\% Engel IA outcome after 24 months. ${ }^{29}$ Centres reporting Engel IA and Engel IA+B (auras not counted) outcomes find $11-14 \%$ more seizure-free patients with the more liberal measure. ${ }^{28} 29$ 
A: evaluations and resective surgeries MTS

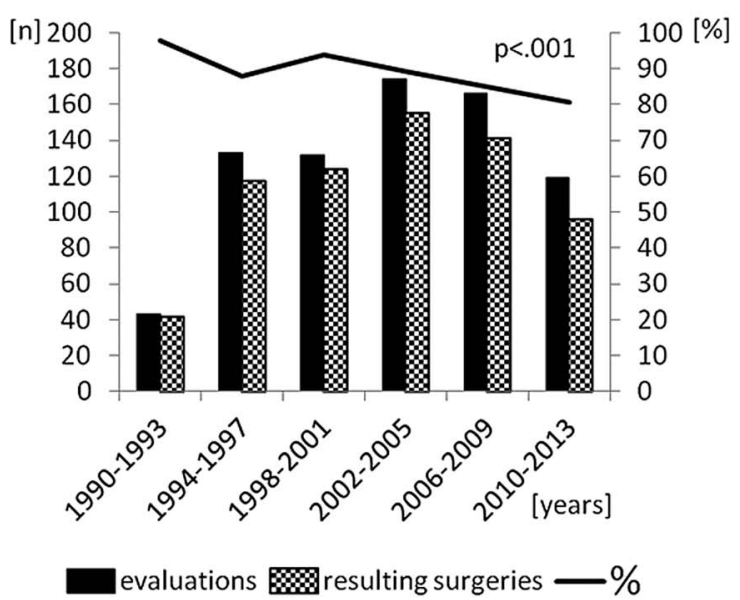

C: temporal lobe resections

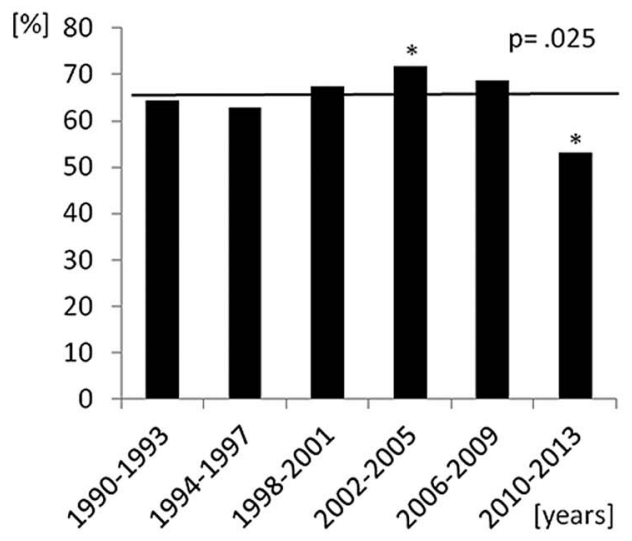

B: evaluations and resective surgeries BT

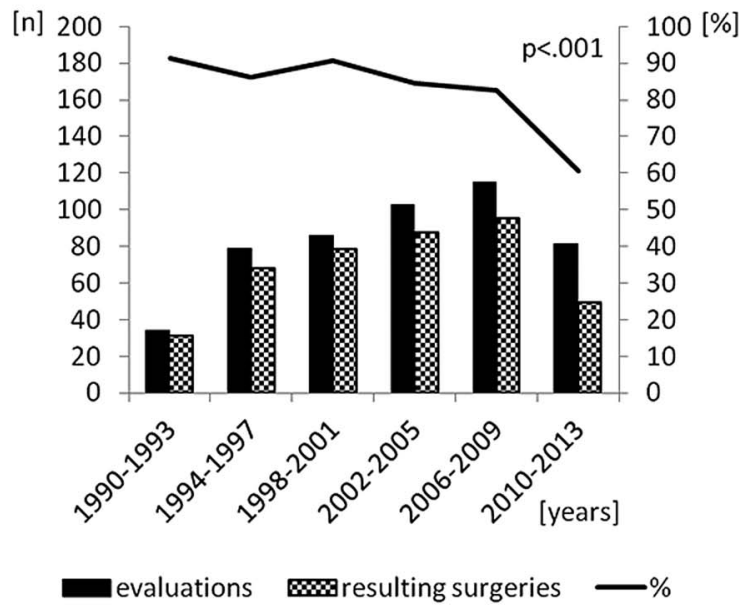

D: invasive recordings/non-lesional

[\%] 45

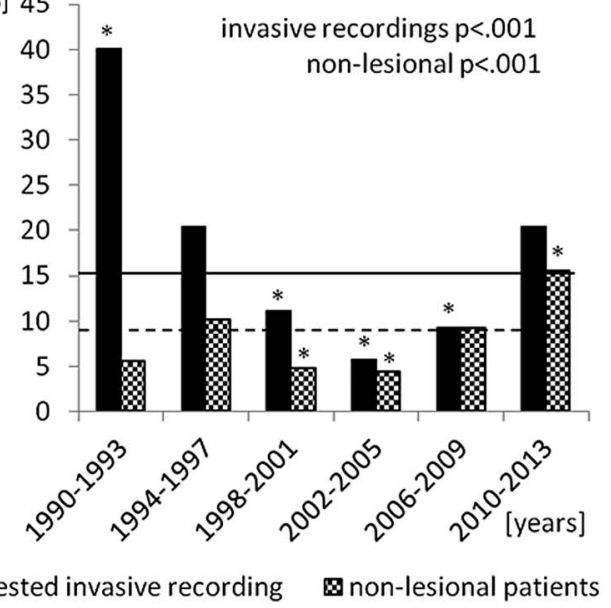

Figure 3 Trends over time: (A and B) evaluation and surgery of MTS and BT over time (C and D) proportion (\%) of (C) temporal lobe resections (total 65.1\%) and (F) suggested invasive recordings (total 15.1\%) and non-lesional patients (total 8.9\%). Legend: $p$ values: test of trend (Mantel-Haenszel $\chi^{2}$ test); * significant deviation from the estimated grand mean $(p<0.05)$ using GLM: solid line: $(A$ and $B) \%$ resulting surgeries $(\%),(C)$ temporal lobe resections (\%), and (D) total suggested invasive recordings (\%) and dotted line: (D) total non-lesional (\%). BT, benign tumours; GLM, generalised linear model; MTS, medial temporal sclerosis.

In comparison to the Bonn series, the rates for MTS and BT were again almost identical to our study: MTS, Bethel 49.4\%/ Bonn 51.8\% and BT, Bethel 61.0\%/Bonn 60.1\%. Patients with FCD showed a divergent outcome: Bethel 45.7\%/Bonn 57.6\%. Another German centre reached an intermediate seizure freedom rate (Engel IA) of 51\% for FCD patients 24 months after surgery. ${ }^{30}$ The highly similar outcomes for MTS and BT suggest that the standard temporal lobe resection types (2/3 resection as preferred in Bethel, or amygdalohippocampectomy, the preferred method in Bonn) and any lesionectomy approach quite safely include the epileptogenic area. In contrast, the less well-demarcated borders of FCD favour a consequent extension of lesionectomy (as in the majority of cases practised in Bonn ${ }^{21}$ and often in Freiburg) ${ }^{30}$ over more circumscribed approaches (as in Bethel until 2010).

There were increasing seizure freedom rates for the total study cohort and the subgroup of FCD and BT (figure 5). A better demarcation of circumscribed epileptogenic lesions due to high-resolution MRI might contribute to this. ${ }^{31}$ Another reason might be the very constant personnel situation at our centre (different from a university setting with more frequent fluctuation), giving the possibility of a constantly learning system. This is especially true for the number of neurosurgeons: in Bethel, three neurosurgeons performed all 2044 resections, whereas the Bonn series named nine neurosurgeons in the author list and the aknowledgements. ${ }^{10}$

As main results, we observed a progressively increasing number of presurgical evaluations and an inverted U-shaped curve of resulting surgical interventions (with a steep initial increase and a slight recent decrease (figures 1 and 2)). This is in line with previous reports on increasing diagnostic facilities, increasing numbers of hospitalisations and video-EEG monitorings but stable surgical numbers. ${ }^{8} 932$ Another study even reported about a slight decrease in surgical numbers. ${ }^{33}$ This increasing gap was related to a declining proportion of patients proceeding to surgery in the smaller centres. ${ }^{8}$ Our series and data from another large German centre ${ }^{10}$ show that the gap between diagnostic and surgical volumes is not restricted to smaller centres. We conclude that a lack of intentness (which might be assumed in small volume centres) alone does not explain this overall gap. We propose the following reasons for this trend: 
Figure 4 Decisions against surgery over time. Legend: $p$ values: for test of trend (Mantel-Haenszel $\chi^{2}$ test); * significant deviation from estimated grand mean $(p<0.05)$ using GLM solid line: $(A, C$ and $D)$ mean patient/parent and $(B)$ mean risk; and dotted line: ( $A, C$ and D) mean neurologist and (B) mean no hypothesis. GLM, generalised linear model.

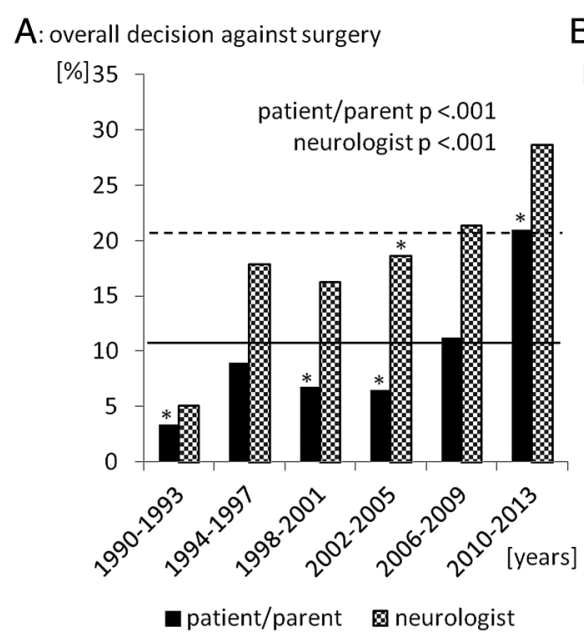

C:decisions against surgery MTS

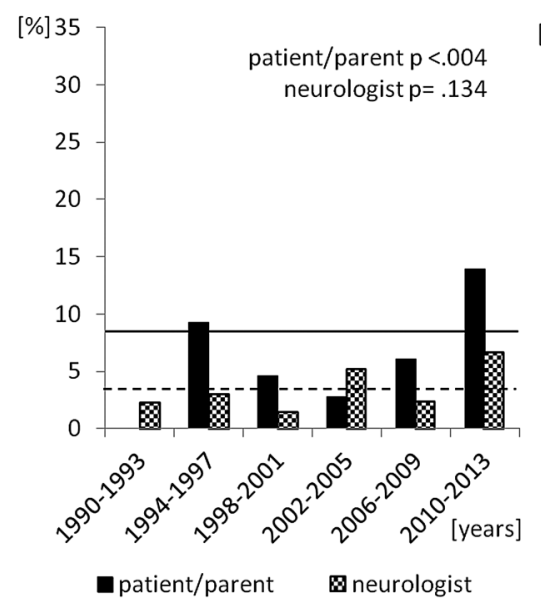

\section{B: neurological reason for rejection} [\%] 35

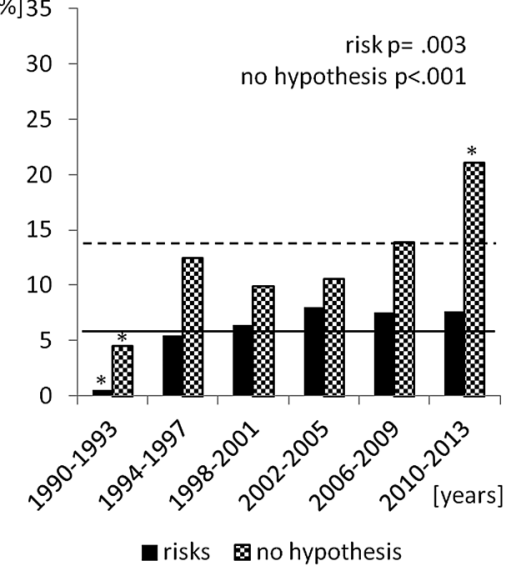

$D$ : decisions against surgery BT

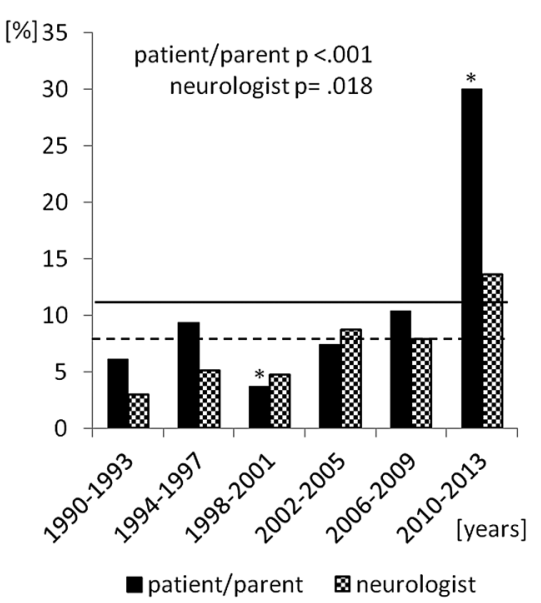

Table 2 Informed consent protocols

\begin{tabular}{|c|c|c|c|c|c|c|}
\hline & & 1990-1996 & $1997-2000$ & 2000-2010 & 2011-2012 & 2013- \\
\hline \multirow{7}{*}{ Neurosurgeons } & Patients/parents & \multicolumn{5}{|l|}{ All } \\
\hline & Extent (pages) & \multicolumn{3}{|l|}{1} & \multicolumn{2}{|l|}{5} \\
\hline & Word count & 158 & 228 & \multirow{2}{*}{309} & \multicolumn{2}{|l|}{2422} \\
\hline & Sketch of procedure & Partly & All & & & \multirow{3}{*}{$\begin{array}{l}\text { Copyright document, } \\
\text { based on legal advice }\end{array}$} \\
\hline & Document type & \multicolumn{4}{|c|}{ Standardised, designed by the neurosurgeon } & \\
\hline & No. of risks detailed & 0 & \multicolumn{3}{|c|}{5.8 (range 4-9), specific risks 1.3 (range $0-3$ ) } & \\
\hline & Information about potentially life-threatening risks & No & \multicolumn{2}{|c|}{$\begin{array}{l}\text { 'Breathing and circulatory } \\
\text { disturbance' }\end{array}$} & 'Vegetative state' & 'Possible death' \\
\hline \multirow[t]{6}{*}{ Epileptologists } & Patients/parents & None & \multicolumn{3}{|c|}{$\begin{array}{l}\text { Partly } \\
\text { Individualised protocol }\end{array}$} & $\begin{array}{l}\text { All } \\
\text { Standardised }\end{array}$ \\
\hline & $\begin{array}{l}\text { Document type } \\
\text { Extent (paqes) }\end{array}$ & $\begin{array}{l}- \\
-\end{array}$ & \multicolumn{2}{|l|}{1} & 2 & $\begin{array}{l}\text { Standardised } \\
4\end{array}$ \\
\hline & Word count & - & \multirow{2}{*}{\multicolumn{2}{|c|}{$327-343$}} & & 838 \\
\hline & Sketch & - & no & & & yes \\
\hline & Anticipated seizure freedom chance in per cent given & - & \multirow{2}{*}{\multicolumn{2}{|c|}{ Yes }} & & \\
\hline & Risks mentioned & - & & No & \multicolumn{2}{|l|}{ Yes } \\
\hline
\end{tabular}

The darker colours indicated increasing degrees of 'intensity'.

1. An increasing proportion of difficult cases as the key reason for this phenomenon. Similar observations and suggestions have been provided by other publications ${ }^{7} 1033$ and are substantiated in our own cohort: first, the proportion of patients with clear indications for surgical treatment decreased over time (figure 3: less patients with MTS, BT and temporal resections). Potential causes are a decreasing incidence of precipitating injuries leading to $\mathrm{MTS}^{6}$ (resulting in a decreasing prevalence after two decades of epilepsy surgery) and an increasing tendency to remove low-grade tumours without formal presurgical epilepsy diagnostics. ${ }^{34}$ Other studies, too, observed a decline in MTS-related epilepsy surgery. ${ }^{6} 1233$ One paper described a doubling of extratemporal resections from 2007 to $2012 .{ }^{9}$ Second, since 2002, we found more non-lesional patients and an increasing need for invasive recordings (figure 3D). Other studies 


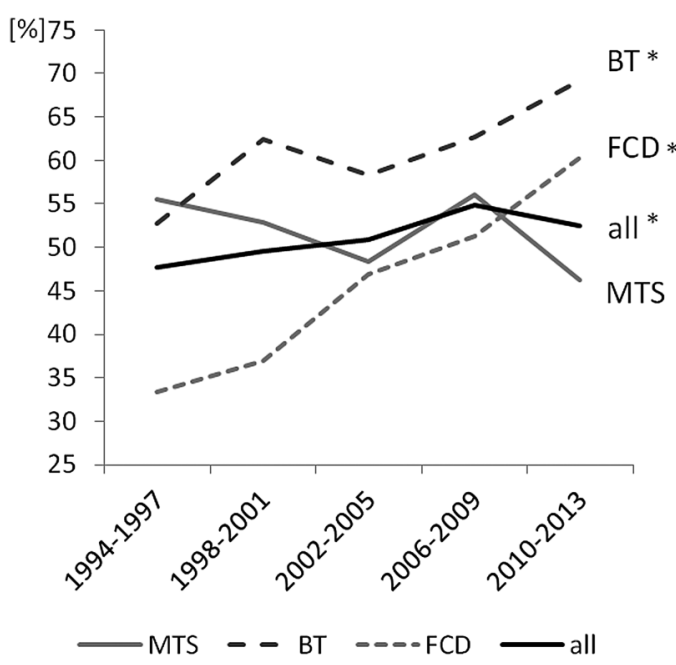

Figure 5 Trends over time: proportion of seizure-free (Engel IA) patients 24 months after surgery (\%). BT, benign tumour; FCD, focal cortical dysplasia 1990-1993 not shown, because of low numbers; MTS, medial temporal sclerosis; * significant test of trend for all periods 1990-2013 (Mantel-Haenszel $\chi^{2}$ test, $\mathrm{p}<0.05$ ).

observed the same trend. ${ }^{9}{ }^{33}$ Jehi et $a l^{33}$ found more patients undergoing invasive recordings without subsequent surgery. There are reports of increasing numbers of MRI negative, non-lesional patients for presurgical evaluation, which also often require intracranial studies.

(1) We observed an increasing rate of decisions against surgery (figure $4 \mathrm{~A}$ ). The higher rejection rate by the neurologist is apparently explained by the above-discussed trend towards more challenging cases for presurgical evaluation. The increasing numbers of patients without a welldelineated epileptogenic zone are in line with this hypothesis and had a stronger impact on decision against surgery than deficit risks (figure 4B). Besides these 'objective' reasons for rejections, there is a trend that more patients opt against surgery (figure 4A, C, D). This is an important and novel explanation for the decline in surgical numbers. Possible reasons are more extended information about chances and risks of surgery (as documented by the increasingly comprehensive information sheets before surgery (table 2)). Recently, the following reasons for patients to step back from surgery were reported: dissatisfaction with the predicted likelihood to become seizure free (52\%), scepticism related to intracranial recordings (51\%) and concerns about surgical risks (14\%). ${ }^{35}$ Another explanation might be fewer patients with a clear upfront motivation for surgical treatment. This may be related to an increasingly early referral for presurgical workup even in patients without explicit surgical motivation. ${ }^{5}$

2. Finally, a backlog of patients amenable for new curative treatment options and its subsequent clear-out may explain the inverted U-shaped curve in newly set up centres in an area without this option before this. Possibly, a later shift of clear cases to centres without special expertise in epilepsy surgery, motivated by the expanding knowledge about the efficacy of surgical treatment for MTS and BT, contributes to the decline of straightforward cases.

Our data do not include information about surgical complications or the frequency of compensation complaints that might influence the decision-making process. In line with other studies, a recent meta-analysis found an overall low risk for permanent neurological deficits in epilepsy surgery due to accidental damage of the brain $(<5 \%){ }^{4}$

\section{CONCLUSION}

Presurgical evaluation has become part of a standard workup for patients with drug-resistant focal epilepsy, even for those without obvious surgically remediable syndromes or without clear surgical motivation at the beginning of the process. This conforms well with the international recommendation to refer patients after failure of two adequately used antiepileptic drugs to an epilepsy centre to check (among other options) the possibility of a potentially curative surgical treatment. ${ }^{5}$ Patients entering the presurgical evaluation more often make the informed choice of not undergoing epilepsy surgery. The increasing capacity of the presurgical programme largely compensates for this trend and the declining number of 'straightforward' cases with MTS or BT. Surgical volumes thus can be maintained. It is encouraging that despite the increase in complicated cases, seizure freedom rates can be kept up and even improved at this centre over time.

Acknowledgements The authors would like to thank the former colleagues contributing to the work of the Bethel epilepsy surgery, namely Drs Lüders, Meencke, Noachtar, Ebner, Hoppe (Neurology), Holthausen, Tuxhorn (Pediatric Neurology), Oppel, Pannek (Neurosurgery), Lahl, Hans (Neuropathology) and Lumiani (Neuroradiology)

Contributors TC involved in collection, analysis and interpretation of the data, and drafting the manuscript and figures. TWM involved in analysis and interpretation of the data, statistical analyses and revision of the manuscript for important intellectual content. IB commented on neuropathological data and revised the manuscript for content. PG participated in collection of clinical data, revision of the manuscript for content and database work. LJH involved in collection of clinical data, database and revision of the manuscript for content. TK provided neurosurgical comments and participated in data collection for outcome and revision of the manuscript for content. MP participated in collection of clinical data, database and revision of the manuscript for content. TP and RS revised the manuscript for important intellectual content. FGW set up structure for data collection on neuroimaging and revised the manuscript for important intellectual content. CGB provided important conceptional influence and revised the manuscript for important conceptual and intellectual content.

Funding The work is supported by the European Union (FP7 DESIRE GA \# 602531 to IB).

Competing interests TWM received financial support from UCB (Monheim, Germany) and Desitin (Hamburg, Germany) for visiting scientific meetings, served on scientific advisory boards and received honoraria for speaking engagements from Eisai (Frankfurt, Germany), UCB and Desitin. RS reports personal fees from UCB (Monheim Germany), outside the submitted work. CGB reports personal fees and other from Eisai (Frankfurt, Germany), UCB (Monheim, Germany) and Desitin (Hamburg, Germany), grants and personal fees from Grifols (Frankfurt, Germany), Diamed (Köln, Germany) and Fresenius Medical Care (Bad Homburg, Germany), outside the submitted work.

Provenance and peer review Not commissioned; externally peer reviewed.

\section{REFERENCES}

1 West S, Nolan SJ, Cotton J, et al. Surgery for epilepsy. Cochrane Database Syst Rev 2015:CD010541.

2 Wiebe S, Blume WT, Girvin JP, et al. A randomized, controlled trial of surgery for temporal-lobe epilepsy. N Engl J Med 2001;345:311-8.

3 Engel J, McDermott MP, Wiebe $S$, et al. Early surgical therapy for drug-resistant temporal lobe epilepsy: a randomized trial. JAMA 2012;307:922-30.

4 Engel J, Wiebe S, French J, et al. Practice parameter: temporal lobe and localized neocortical resections for epilepsy: report of the Quality Standards Subcommittee of the American Academy of Neurology, in association with the American Epilepsy Society and the American Association of Neurological Surgeons. Neurology 2003:60:538-47.

5 Kwan P, Arzimanoglou A, Berg AT, et al. Definition of drug resistant epilepsy: consensus proposal by the ad hoc Task Force of the ILAE Commission on Therapeutic Strategies. Epilepsia 2010;51:1069-77.

6 Helmstaedter C, May TW, von Lehe M, et al. Temporal lobe surgery in Germany from 1988 to 2008: diverse trends in etiological subgroups. Eur J Neurol $2014 ; 21: 827-34$ 
7 Neligan A, Haliasos N, Pettorini B, et al. A survey of adult and pediatric epilepsy surgery in the United Kingdom. Epilepsia 2013;54:e62-5.

8 Englot DJ, Ouyang D, Garcia PA, et al. Epilepsy surgery trends in the United States, 1990-2008. Neurology 2012;78:1200-6.

9 Kaiboriboon K, Malkhachroum AM, Zrik A, et al. Epilepsy surgery in the United States: analysis of data from the National Association of Epilepsy Centers. Epilepsy Res 2015;116:105-9.

10 Bien CG, Raabe AL, Schramm J, et al. Trends in presurgical evaluation and surgical treatment of epilepsy at one centre from 1988-2009. J Neurol Neurosurg Psychiatr 2013;84:54-61.

11 Carlson C. The changing face of epileptology? Results of the initial Q-PULSE survey. Epilepsy Curr Am Epilepsy Soc 2013;13:305-7.

12 Van Gompel JJ, Ottman R, Worrell GA, et al. Use of anterior temporal lobectomy for epilepsy in a community-based population. Arch Neurol 2012;69:1476-81.

13 Carreno M, Lüders HO. General principles of presurgical evaluation. In: Lüders HO, ed. Textbook of epilepsy surgery. London: Informa, 2008:409-22.

14 Palmini A, Najm I, Avanzini G, et al. Terminology and classification of the cortical dysplasias. Neurology 2004;62:S2-8.

15 Blümcke I, Thom M, Aronica E, et al. The clinicopathologic spectrum of focal cortical dysplasias: a consensus classification proposed by an ad hoc Task Force of the ILAE Diagnostic Methods Commission. Epilepsia 2011;52:158-74.

16 Galaburda AM, Sherman GF, Rosen GD, et al. Developmental dyslexia: four consecutive patients with cortical anomalies. Ann Neurol 1985;18:222-33.

17 Akil M, Lewis DA. Cytoarchitecture of the entorhinal cortex in schizophrenia. Am J Psychiatry 1997;154:1010-2.

18 Binder DK, Schramm J. Transsylvian functional hemispherectomy. Childs Nerv Syst ChNS Off I Int Soc Pediatr Neurosurg 2006;22:960-6.

19 Engel J Jr, Cascino GD, Shields WD. Surgically remediable syndromes. In: Engel J, Jr., Pedley TA, eds. Epilepsy: a comprehensive textbook. Philadelphia (PA): Wolters Kluwer-Lippincott Williams \& Wilkins, 1761-9.

20 Krsek P, Maton B, Jayakar P, et al. Incomplete resection of focal cortical dysplasia is the main predictor of poor postsurgical outcome. Neurology 2009;72:217-23.

21 Wagner J, Urbach H, Niehusmann P, et al. Focal cortical dysplasia type llb: completeness of cortical, not subcortical, resection is necessary for seizure freedom. Epilepsia 2011;52:1418-24.
22 Elsharkawy AE, Pannek H, Woermann FG, et al. Apical temporal lobe resection; 'tailored' hippocampus-sparing resection based on presurgical evaluation data. Acta Neurochir (Wien) 2011;153:231-8.

23 Engel J Jr, Van Ness PC, Rasmussen TB, et al. Outcome with respect to epileptic seizures. In: Engel J Jr, ed. Surgical treatment of the epilepsies. New York: Raven Press, 1993:609-21.

24 McIntosh AM, Wilson SJ, Berkovic SF. Seizure outcome after temporal lobectomy: current research practice and findings. Epilepsia 2001:42:1288-307.

25 Jobst BC, Cascino GD. Resective epilepsy surgery for drug-resistant focal epilepsy: a review. JAMA 2015:313:285-93.

26 Varadkar S, Bien CG, Kruse CA, et al. Rasmussen's encephalitis: clinical features, pathobiology, and treatment advances. Lancet Neurol 2014;13:195-205.

27 Hemb M, Velasco TR, Parnes MS, et al. Improved outcomes in pediatric epilepsy surgery: the UCLA experience, 1986-2008. Neurology 2010;74:1768-75.

28 Spencer SS, Berg AT, Vickrey BG, et al. Predicting long-term seizure outcome after resective epilepsy surgery: the multicenter study. Neurology 2005;65:912-8.

29 de Tisi J, Bell GS, Peacock JL, et al. The long-term outcome of adult epilepsy surgery, patterns of seizure remission, and relapse: a cohort study. Lancet 2011;378:1388-95.

30 Fauser S, Essang C, Altenmüller DM, et al. Long-term seizure outcome in 211 patients with focal cortical dysplasia. Epilepsia 2015;56:66-76.

31 Winston GP, Micallef $C$, Kendell $B E$, et al. The value of repeat neuroimaging for epilepsy at a tertiary referral centre: 16 years of experience. Epilepsy Res 2013;105:349-55.

32 Schiltz NK, Koroukian SM, Lhatoo SD, et al. Temporal trends in pre-surgical evaluations and epilepsy surgery in the U.S. from 1998 to 2009. Epilepsy Res 2013;103:270-8.

33 Jehi L, Friedman D, Carlson C, et al. The evolution of epilepsy surgery between 1991 and 2011 in nine major epilepsy centers across the United States, Germany, and Australia. Epilepsia 2015;56:1526-33.

34 Jakola AS, Myrmel KS, Kloster R, et al. Comparison of a strategy favoring early surgical resection vs a strategy favoring watchful waiting in low-grade gliomas. JAMA 2012;308:1881-8.

35 Fois C, Kovac S, Khalil A, et al. Predictors for being offered epilepsy surgery: 5-year experience of a tertiary referral centre. J Neurol Neurosurg Psychiatr 2016;87:209-11. 